\title{
Comprehensive Analysis of DNA Methylation Regulator DNA Methyltransferase (DNMT) Family and Ten-Eleven-Translocation (TET) Enzymes Family in Pan-Cancer
}

\section{Cheng Ouyang}

The First Affiliated Hospital of China Medical University

Hao Li

The First Affiliated Hospital of China Medical University

Liping Sun ( $\square$ lpsun@cmu.edu.cn )

The First Affiliated Hospital of China Medical University https://orcid.org/0000-0003-3637-0305

\section{Research}

Keywords: DNA methylation, DNMT, TET enzymes, pan-cancer, prognosis

Posted Date: January 29th, 2021

DOl: https://doi.org/10.21203/rs.3.rs-154338/v1

License: (c) (1) This work is licensed under a Creative Commons Attribution 4.0 International License.

Read Full License 


\section{Abstract}

Background: DNA methyltransferase (DNMT) family and ten-eleven-translocation (TET) family enzymes play pivotal roles in regulating DNA methylation, and are closely related to diverse cancers. This study was designed to clarify the specific roles of DNMT and TET genes in pan-cancers.

Methods: The expression, mutation, copy number variations (CNVs), cancer-related pathways, immune cell infiltration correlation, and prognostic potential of DNMT/TET genes were systematically investigated in 33 cancer types using next-generation sequence data from the Cancer Genome Atlas database.

Results: DNMT3B was more highly expressed in the majority of tumors analyzed than in normal tissues. Most DNMT/TET genes were frequently mutated in uterine carcinosarcoma, and TET1 and TET2 showed higher mutation frequencies in various cancer types. DNMT3B exhibited inclusive copy number amplification in almost all cancers, such as stomach adenocarcinoma(STAD) and colon adenocarcinoma(COAD)I, while most DNMT/TET genes displayed highly copy number deletion in kidney chromophobe $\mathbb{K} \mathrm{KICH} \otimes$. DNMT/TET genes were mainly involved in the following cancer-related pathways: UV response DN, mitotic spindle, cholesterol homeostasis, TGF beat signaling, xenobiotic metabolism, G2/M checkpoint, and E2F targets. DNMT/TET genes were significantly correlated with NK cells, CD4 positive T cells, and Tfh cells. Additionally, Most DNMT/TET genes were significantly associated with lower survival rates of adrenocortical carcinoma (ACC), mesothelioma, and liver hepatocellular carcinoma (LIHC), but played a protective role in thymoma (THYM). Furthermore, overexpression of most DNMT genes, except for DMAP1, was associated worse prognoses in pan-cancer.

Conclusion: These results suggest that DNMT/TET genes can serve as potential predictors for prognosis and treatment in pan-cancer, providing new insight for future study.

\section{Background}

Traditionally, it was believed that cancer is caused by genetic alterations including deletions, mutations, genomic instability, copy number variations (CNVs), insertions, recombination, and single-nucleotide polymorphisms [1]. However, emerging evidence suggests that genetic and epigenetic changes interact in the occurrence, development, and recurrence of cancer $[2,3]$. Studies show that half of the tumor suppressor gene inactivation in cancer is the result of epigenetic rather than genetic mechanisms [4]. In recent years, the development of high-throughput sequencing has led to a drastic increase in genetic and epigenetic research in diverse cancers.

DNA methylation is one of the most important epigenetic markers, and is involved in parental imprinting, DNA replication, regulation of gene expression, $\mathrm{X}$ chromosome inactivation, and stable gene silencing [1, $5,6]$. DNA methylation and demethylation are dynamically regulated by the interplay between DNA methyltransferase (DNMT) and ten-eleven-translocation (TET) enzyme families [7-9]. DNA methylation and demethylation are mutually transformable processes, and are respectively catalyzed by methyltransferase and demethyltransferase. DNMT genes are responsible for transferring a methyl group 
to the fifth carbon of a cytosine residue to form 5-methylcytosine $(5 \mathrm{mC})$ and mediating active DNA methylation [10]. TET enzymes oxidize $5 \mathrm{mC}$ to form 5-hydroxymethylcytosine ( $5 \mathrm{hmC}), 5$-formylcytosine $(5 \mathrm{fC})$, and 5-carboxylcytosine (5caC), thereby regulating active DNA demethylation [11-15].

Accumulating evidence suggests a close association between epigenetic changes and the onset and progression of multiple tumor types. DNA methylation plays a critically important in activating or silencing gene transcription [16-18]. Methylation of promoter region $\mathrm{CpG}$ islands is important in transcriptional regulation, and leads to tumor suppressor gene silencing in carcinogenesis $[17,19]$. DNMT/TET gene activity plays a pivotal role in the dynamic regulation of DNA methylation patterns [20, 21], which is essential in tumorigenesis [2]. Abnormal methylation silences the secreted frizzled-related protein (SFRP) gene family, activating the classic Wnt pathway, and influencing the occurrence and development of colorectal cancer [22]. In breast cancer, mir-22 inhibits TET genes expression, resulting in loss of function. This leads to increased promoter region methylation, tumor development, and metastasis [23].

The past decade had seen increasingly rapid advances in the field of DNA methylation in tumorigenesis. However, the specific roles of DNMT/TET genes in tumorigenesis have not been fully elucidated. Given the role of epigenetic changes in cancer, it is important to understand the expression, mutations, and CNVs in DNA methylation regulators like the DNMT/TET genes and their prognostic potential. This study was designed to analyze DNMT/TET genes data from 33 types of cancers in The Cancer Genome Atlas (TCGA). We postulate that this work will generate fresh insight into the way DNMT/TET genes influence cancer.

\section{Materials And Methods}

\section{Collection of DNMT/TET genes}

We collected six key DNMT family members and three key TET family members [13-15] from published review papers. We manually converted the gene symbols into Human Genome Organisation Gene Nomenclature Committee (HGNC) symbols and Ensemble gene IDs from GeneCards. (https://www.genecards.org/)

\section{Pan-cancer genome-wide omics data from next-generation sequence data}

We obtained omics datasets from TCGA database (http://cancergenome.nih.gov/), aimed at TPM (Transcripts Per Kilobase Million) expression, mutation, CNVs, and clinical information (survival status, survival time) from 33 different types of cancers, including $\mathrm{CHOL}$, cholangiocarcinoma; KIRC, kidney renal clear cell carcinoma; DLBC, lymphoid neoplasm diffuse large b-cell lymphoma; KIRP, kidney renal papillary cell carcinoma; UVM, uveal melanoma; KICH, kidney chromophobe; MESO, mesothelioma; LGG, brain lower grade glioma; THYM, thymoma; GBM, glioblastoma multiforme; TGCT, testicular germ cell tumors; BRCA, breast cancer; ESCA, esophageal carcinoma; LUSC, lung squamous cell carcinoma; PAAD, pancreatic adenocarcinoma; LUAD, lung adenocarcinoma; READ, rectum adenocarcinoma; LAML, acute 
myeloid leukemia; COAD, colon adenocarcinoma; SARC, sarcoma; UCS, uterine carcinosarcoma; PCPG, pheochromocytoma and paraganglioma; UCEC, uterine corpus endometrial carcinoma; ACC, adrenocortical carcinoma; OV, ovarian serous cystadenocarcinoma; CESC, cervical squamous cell carcinoma and endocervical adenocarcinoma; HNSC, head and neck squamous carcinoma; LIHC, liver hepatocellular carcinoma; THCA, thyroid carcinoma; BLCA, bladder urothelial carcinoma; PRAD, prostate adenocarcinoma; SKCM, skin cutaneous melanoma; STAD, stomach adenocarcinoma. All of the TCGA data were download via UCSC XENA (https://xenabrowser.net/).

\section{Analysis of differentially expressed genes in pan-cancer}

Using the Deseq2 package in $R$, we identified differential DNMT/TET genes expression in pan-cancer, using |Log2 Fold Changel $>1$ and adjusted P-values $<0.05$. TBtools software was used to draw a heatmap.[24]

\section{Protein-wide omics data across 20 cancer types from protein expression data}

Protein expression data were derived from Human Protein Atlas Datasets (https://www.proteinatlas.org/), DNMT/TET protein expression were systematically analyzed in twenty-one cancer types, including SKCA, BRCA, ENCA, RACA, STCA, PACA, carcinoid, OV, URCA, LIHC, CECA, THCA, melanoma, PRCA, COCA, lymphoma, TECA, glioma, LUCA, HNSC. The results were visualized by GraphPad Prism version 8 (GraphPad Software, La Jolla, CA, USA).

\section{Genome-wide mutation and CNVs in DNMT/TET genes across pan-cancer}

Mutation frequencies and CNVs were calculated. Using GraphPad Prism software, mutation and TPM expression were analyzed by Mann-Whitney $\mathrm{U}$ test, and the correlation between CNVs and gene expression was analyzed using the Kruskal-Wallis test. $\mathrm{P}<0.05$ was considered to be statistically significant.

\section{Correlation between DNMT/TET genes, oncogenic pathway activity, and immune cell infiltration}

The correlation between gene expression and cancer-related pathways were determined using Gene Set Variation Analysis (GSVA). This is a gene set enrichment (GSE) method to estimate pathway activity variation based on a sample expression matrix in an unsupervised, non-parametric manner. The Spearman Correlation Coefficient (SCC) was calculated to assess the correlation between gene expression and pathway activity, and immune cell infiltration. $|\mathrm{SCC}|>0.25$ and adjusted P-value $<0.05$ were considered to be a significantly correlation. The results were visualized by Cytoscape v3.7.1.

\section{Clinical significance of DNMT/TET genes}

The median expression of each gene was selected as a cut-off value to divide patients into two groups. Kaplan-Meier analysis, with the log-rank test, was used to compare survival rates between the two groups. Additionally, gene expression and prognostic value associations were analyzed using the GEPIA2(Gene 
Expression Profiling Interactive Analysis 2) database. P-value $<0.05$ was considered as statistically significant.

\section{Results}

\section{DNMT/TET genes expression profiles in pan-cancer}

We summarized the chromosomal positions of all DNMT/TET family members across the published literature (Table 1). We then analyzed the gene expression differences in TCGA. The results showed heterogeneous expression of DNTM and TET genes in different cancer types. DNMT3B was highly expressed in the majority of tumors; DNMT3L was expressed in low levels in $\mathrm{CHOL}, \mathrm{KICH}, \mathrm{KIRP}$, and KIRC, and had increased expression in UCEC READ, ESCA, and LUSC. (Figure 1a). The Log2FC and adjusted Pvalue of the results are listed in Supplementary Table 1. We visualized the differential expression of DNMT3B in different cancers (Figure 1b). DNMT3B expression was up regulated in many cancer types, including BLCA, BRCA, CESC, CHOL, COAD, ESCA, GBM, HNSC, KIRC, KIRP, LIHC, LUAD, LUSC, PRAD, READ, STAD and UCEC.

Table 1 Basic characteristics of DNMT/TET genes 


\begin{tabular}{|c|c|c|c|c|c|}
\hline HGNC ID (gene) & GENE ID & Approved symbol & Synonym(s) & Exon & Chromosomal location \\
\hline \multirow[t]{7}{*}{2976} & \multirow[t]{7}{*}{1786} & \multirow[t]{7}{*}{ DNMT1 } & AIM & \multirow[t]{7}{*}{41} & \multirow[t]{7}{*}{ 19p13.2 } \\
\hline & & & DNMT & & \\
\hline & & & MCMT & & \\
\hline & & & CXXC9 & & \\
\hline & & & HSN1E & & \\
\hline & & & ADCADN & & \\
\hline & & & m.Hsal & & \\
\hline \multirow[t]{5}{*}{2977} & \multirow[t]{5}{*}{1787} & \multirow[t]{5}{*}{ TRDMT1 } & DMNT2 & \multirow[t]{5}{*}{15} & \multirow[t]{5}{*}{$10 \mathrm{p} 13$} \\
\hline & & & DNMT2 & & \\
\hline & & & PUMET & & \\
\hline & & & RNMT1 & & \\
\hline & & & MHSAIIP & & \\
\hline \multirow[t]{4}{*}{2978} & \multirow[t]{4}{*}{1788} & \multirow[t]{4}{*}{ DNMT3A } & TBRS & \multirow[t]{4}{*}{34} & \multirow[t]{4}{*}{$2 \mathrm{p} 23.3$} \\
\hline & & & HESJAS & & \\
\hline & & & DNMT3A2 & & \\
\hline & & & M.HsallIA & & \\
\hline \multirow[t]{3}{*}{2979} & \multirow[t]{3}{*}{1789} & \multirow[t]{3}{*}{ DNMT3B } & ICF & \multirow[t]{3}{*}{24} & \multirow[t]{3}{*}{$20 q 11.21$} \\
\hline & & & ICF1 & & \\
\hline & & & M.HsallIB & & \\
\hline 2980 & 29947 & DNMT3L & - & 12 & $21 q 22.3$ \\
\hline \multirow[t]{5}{*}{18291} & \multirow[t]{5}{*}{55929} & \multirow[t]{5}{*}{ DMAP1 } & EAF2 & \multirow[t]{5}{*}{11} & \multirow[t]{5}{*}{$1 p 34.1$} \\
\hline & & & SWC4 & & \\
\hline & & & MEAF2 & & \\
\hline & & & DNMAP1 & & \\
\hline & & & DNMTAP1 & & \\
\hline \multirow[t]{3}{*}{29484} & \multirow[t]{3}{*}{80312} & \multirow[t]{3}{*}{ TET1 } & LCX & \multirow[t]{3}{*}{20} & \multirow[t]{3}{*}{$10 q 21.3$} \\
\hline & & & CXXC6 & & \\
\hline & & & bA119F7.1 & & \\
\hline
\end{tabular}




\begin{tabular}{|c|c|c|c|c|c|}
\hline \multirow[t]{2}{*}{25941} & \multirow[t]{2}{*}{54790} & \multirow[t]{2}{*}{ TET2 } & MDS & \multirow[t]{2}{*}{15} & \multirow[t]{2}{*}{$4 q 24$} \\
\hline & & & KIAA1546 & & \\
\hline \multirow[t]{2}{*}{28313} & \multirow[t]{2}{*}{200424} & \multirow[t]{2}{*}{ TET3 } & BEFAHRS & \multirow[t]{2}{*}{17} & \multirow[t]{2}{*}{$2 p 13.1$} \\
\hline & & & hCG_40738 & & \\
\hline
\end{tabular}

Furthermore, we analyzed DNMT/TET protein expression levels in different cancers types. In COAD, STCA, LIHC and PACA, DNMT3A, TRDMT1, and TET2 were high expressed, while TET3 expression was significantly reduced (Figure2a). Detailed DNMT/TET protein expression information in 21 cancers is shown in Supplementary Table 2. DNMT3B protein expression in different human cancers is shown in Figure 2b.

Additionally, we elucidated the correlations between DNMT and TET genes expression in pan-cancer. Significant correlations were identified between DNMT3A-TET1 $(r=0.57, p<0.05)$, TET2-TET3 $(r=0.55$, $p<0.05)$, and TET1-TET3 ( $r=0.50, p<0.05)$ (Figure3).

\section{DNMT/TET genetic alterations in pan-cancer}

We examined the DNMT/TET gene mutation frequency in 33 cancer types. Most DNMT/TET genes were frequently mutated in UCEC, and rare mutations were observed in CHOL, PCPG, and THCA. TET1 and TET2 had higher mutation frequencies than did other genes in pan-cancer, and the overall average mutation frequency of DNMT/TET genes in different cancer types ranged from $0 \%$ to $34.9 \%$ (Figure 4a). We also investigated DNMT/TET gene CNVs in pan-cancer (Figure 4b). DNMT3B exhibited inclusive copy number amplification in STAD, COAD, READ, BLCA, ESCA, and LUSC, but almost no copy number deletions in multiple cancer types. Most DNMT/TET genes displayed copy number deletions in $\mathrm{KICH}$, but copy number variations were rarely observed in LIHC and LAML.

Next, we analyzed the relationship between DNMT/TET gene mutations and expression levels in human cancers and visualized the statistically significant results (Figure 5). DNMT1 expression level changes were related to its mutation in COAD, ESCA, STAD, UCEC, and PRAD. Similarly, mutations in DNMT3L and TET2 also affected their expression levels in COAD. The details were shown in Supplementary Table 3. The association between CNVs and DNMT/TET gene expression levels in multiple cancer types is shown in Figure 6. We found that almost all expression changes in DNMT/TET genes were associated with CNVs.

\section{The correlation between DNMT/TET genes and cancer-related pathways}

We investigated the correlation between DNMT/TET gene expression and cancer related pathways to clarify the molecular significance of DNMT/TET genes in carcinogenesis. DNMT/TET genes were significantly correlated with multiple carcinogenic pathways, playing both activation and suppression roles (Figure 7a). We concluded that DNMT/TET genes are mainly involved in the following cancerrelated pathways: UV response DN, mitotic spindle, cholesterol homeostasis, TGF beta signaling, 
xenobiotic metabolism, G2/M checkpoint, and E2F targets (Supplementary Table4). We also found that DNMT3A, TET1, TET2, and TET3 were more likely to be related to the tumor occurrence and progression pathways (Figure 7b).

\section{The correlation between DNMT/TET genes and immune infiltration}

The correlation between DNMT/TET gene expression and immune cell infiltration in pan-cancer were assessed. DNMT/TET genes were significantly correlated with NK cells, CD4 positive T cells, and Tfh cells. Notably, the correlation between DNMT/TET genes and immune cell infiltration tended to be positive. In particular, TET2, TRDMT1, and TET3 showed more stronger correlation. (Figure 8)

\section{Prognostic value of DNMT/TET genes in pan-cancer}

The association between DNMT/TET gene expression and prognosis in patients with different cancers is shown in Figure 9a. Most DNMT/TET genes were significantly associated with lower survival rates in ACC, MESO, and LIHC, but played a protective role in THYM. DNMT3B was related to poor prognosis in many cancers, including ACC, KIRC, KIRP, LGG, LIHC, MESO, SARC, and UCEC. TRDMT1 and DNMT3A had clearly different effects on prognosis in various cancer types (Figure 9b). In ACC, KICH, LIHC, PRAD, and SARC, TRDMT1 was a predictor of poor prognosis but not in COAD, GBM, KIRC, PCPG, and READ. Meanwhile, DNMT3A expression was associated with poor prognosis in ACC, KICH, LGG, LIHC, and MESO, but not in LAML, PAAD, THYM, and UCS. Moreover, high or low DNMT gene expression was statistically significantly related to patient survival status (Figure 10). Increased DNMT gene expression, except for DMAP1, was associated worse prognoses in pan-cancer. Compared to other genes, DNMT3B had the worst prognosis in cancer patients. No significant correlation was observed between the expression levels of TET1 or TET2 and overall cancer survival.

\section{Discussion}

Previous studies verified an intimate relationship between DNMT/TET genes and cancers. However, the potential implication of these genes in pan-cancer is not well studied. To illustrate the pivotal role of DNMT and TET genes in cancer, we performed a thorough pan-cancer analysis of the expression and transcriptome patterns of core DNMT and TET genes using data obtained from TCGA. Our results show that expression of DNMT/TET genes is heterogeneous in certain cancers and paracancer tissues, and affects patient prognosis. The identified correlation between DNMT/TET gene expression, immune cell infiltration, and cancer-related pathways highlights the significance of DNMT/TET genes in tumorigenesis.

Our systematic analysis of TPM data of nine key DNMT/TET genes from TCGA database provides an overview of DNMT/TET gene expression profiles in 33 human cancer types. DNMT/TET gene expression in different cancer types was heterogeneous and DNMT3B was expressed in most cancer types. A review of the available literature supports our results, showing that expression of DNMT genes is increased in a host of malignant tumors, including PRCA, leukemia, BRCA, PAAD, and COCA [25]. Additionally, TET genes 
expression is substantially decreased in BRCA, LIHC, LUCA, PAAD, and PRCA [26]. DNMT3B expression was increased in BRCA, [27] PRCA, [28] and COAD[29]. We found that DNMT3L expression levels were increased in COAD, READ, and LUCA, and decreased in CHOL, PRAD, and BLCA. These results are consistent with those showing that DNMT3L is highly expressed in LUCA [30] and gastrointestinal stromal tumor[31]. It is worth noting that our findings show that overexpression of DNMT3B and DNMT3L may have important impacts on numerous cancers. The underlying mechanisms of DNMT3B and DNMT3L in cancer deserve further investigation, and may provide new ideas for cancer treatment.

DNMT or TET genes mutations may be responsible for the occurrence and progression of tumors [32, 33]. Through analyzing DNMT/TET genes mutations in multiple cancers we found that the total average mutation frequency ranged from $0 \%$ to $34.9 \%$, and that TET1 and TET2 had relatively high mutation frequencies in pan-cancer. TET genes were frequently mutated in various cancers. TET genes mutations were previously detected in hematopoietic malignancies, melanoma, and some solid tumors [15, 34]. TET2 mutations were frequently detected in myeloid malignancies ( 15\%) [35]. Moreover, genetic studies have shown that TET2 is essential for the self-renewal and differentiation of hematopoietic stem cells in mouse models, and have confirmed that TET2 inactivation leads to the development of myeloid malignancies [36-38]. TET2 mutation has been detected in AML, CMML, MPD, and MDS [39], and TET2 mutation frequency is significantly higher than that of TET1 and TET3. In solid tumors, TET genes mutations were observed in COCA [40] and CCRCC [41]. Interestingly, most DNMT/TET genes showed high mutation frequencies in UCEC, which is a cancer type considered to have high global mutation burden [42]. DNMT/TET genes mutations were rare in CHOL, PCPG, and THCA. Furthermore, TET genes usually functioned as cancer suppressors. Indeed, TET genes were reported to be targets of oncogenic miRNAs [23], and reduced TET genes expression is often detected in cancer cells [26]. Furthermore, TET genes mutations may interfere with TET genes expression, which plays a pivotal role in human tumors $[43,44]$. Indeed, our results show that TET2 and TET3 mutations were significantly related to their expression in some cancers.

Sporadic CNVs significantly affect genomic stability in cancer, and CNVs are observed in nearly $80 \%$ of cancers $[45,46]$. We noticed that DNMT3B exhibited extensive CNV in different cancers and almost all DNMT/TET genes showed copy number deletion in $\mathrm{KICH}$. Additionally, significant copy number changes were usually related to alterations in the expression of the corresponding genes [47]. We found that CNVs and DNMT/TET genes expression levels were associated in multiple cancer types. Together, these findings strongly suggest that genetic changes might contribute to DNMT/TET genes expression changes in carcinogenesis. The potential regulation mechanism related to this deserves further exploration.

We found that most DNMT/TET genes were positively correlated with each other, especially DNMT3ATET1, TET2-TET3, and TET1-TET3. DNMT/TET genes might have a synergistic effect in tumorigenesis, although additional investigation is necessary to determine the mechanism of interaction between these genes in oncogenesis. 
Our results indicate that DNMT/TET genes expression is significantly associated with multiple cancerrelated pathways, especially in UV response DN, mitotic spindle, cholesterol homeostasis, TGF beat signaling, xenobiotic metabolism, G2/M checkpoint, and E2F targets. Individual DNMT/TET genes presented different associations with distinct cancer-related pathways, suggesting that each DNMT/TET gene may have different functions. These results are consistent with those of a previous study showing that DNMT/TET genes have distinct expression patterns and functions $[9,48]$. The methylation of mouse oocyte genome was less than $50 \%$, which is significantly lower than that of the sperm genome, and is related to DNMT1 cytoplasmic retention [49, 50]. TET1 was highly expressed in mouse ESCs [101]. Unlike TET1, TET3 expression was mainly confined to oocytes and zygotes, where it appeared to contribute to the active demethylation or conversion of $5 \mathrm{mC}$ to $5 \mathrm{hmc}$ in male prokaryotes after fertilization [51]. Further investigations are required to analyze the common and diverse functions of these genes in tumorigenesis.

Analysis of the relationship between pan-oncogene expression and immune cell infiltration showed that NK, CD $4^{+}$T, and Tfh cells were most significantly associated with DNTM/TET genes, and with TET2, TRDMT1, and TET3 in particular. The TET protein family has an important influence on maintaining immune system homeostasis by driving Treg cells, and that changes in this may result in the occurrence of cancers [52, 53]. Another study suggested that TET2 regulates Th1 cells, playing a pivotal role in prevention of excessive inflammation in experimental autoimmune encephalomyelitis (EAE) [54]. Together, these findings suggest that further study of DNMT/TET genes may provide novel anti-cancer therapeutics.

Lastly, we evaluated the value of DNMT/TET genes in pan-cancer prognosis. Most DNMT/TET genes were correlated with poor prognosis in ACC, MESO, and LIHC, and were correlated with good prognosis in THYM. Survival analysis also showed that high DNMT genes expression, except for DMAP1, was significantly associated with poor prognosis in pan-cancer and that TET1 and TET2 showed no statistical significance with overall survival in pan-cancer. Early studies showed that DNMT genes were poor prognostic factors in diverse cancers [14], including AML [55], STAD [56], and LUCA [57]. More interestingly, our results revealed that TRDMT1 and DNMT3A showed distinctly disparate prognoses in pan-cancer. DNMT3A expression is related to poor survival rate in hematologic cancers [58], and CMML [59], and in AML DNMT3A acts as a tumor suppressor [60]. This could be explained by different DNMT3A expression patterns in different cancers. Taken together, these findings suggest that DNMT genes can serve as prognostic predictors in pan-cancer. Future studies are needed to reveal the critical role of DNMT/TET genes in the prognosis of various tumors.

\section{Conclusion}

The heterogeneous expression of DNMT/TET genes in pan-cancer is closely related to their mutation, CNVs, and cancer-related pathways, including UV response DN, mitotic spindle, cholesterol homeostasis, TGF beta signaling, xenobiotic metabolism, G2/M checkpoint, and E2F targets. These expression levels of DNMT/TET genes are also relevant to immune infiltrating cells, including NK cells, CD4 positive T cells, 
and Tfh cells. Furthermore, DNMT/TET genes could serve as prognostic indicators, and might provide new insights for future studies.

\section{Abbreviations}

TCGA: The Cancer Genome Atlas

GEPIA2: Gene Expression Profiling Interactive Analysis 2

HGNC: Human Genome Organisation Gene Nomenclature Committee

GSVA: Gene Set Variation Analysis

SCC: Spearman Correlation Coefficient

TPM: Transcripts Per Kilobase Million

CNVs: Copy Number Variations

DNMT: DNA methyltransferase

TET: ten-eleven-translocation

5mC: 5-methylcytosine

5hmC: 5-hydroxymethylcytosine,

5fC: 5-formylcytosine

5caC: 5-carboxylcytosine

$\mathrm{CHOL}$, cholangiocarcinoma; KIRC, kidney renal clear cell carcinoma; DLBC, lymphoid neoplasm diffuse large b-cell lymphoma; KIRP, kidney renal papillary cell carcinoma; UVM, uveal melanoma; KICH, kidney chromophobe; MESO, mesothelioma; LGG, brain lower grade glioma; THYM, thymoma; GBM, glioblastoma multiforme; TGCT, testicular germ cell tumors; BRCA, breast cancer; ESCA, esophageal carcinoma; LUSC, lung squamous cell carcinoma; PAAD, pancreatic adenocarcinoma; LUAD, lung adenocarcinoma; READ, rectum adenocarcinoma; $L A M L$, acute myeloid leukemia; $C O A D$, colon adenocarcinoma; SARC, sarcoma; UCS, uterine carcinosarcoma; PCPG, pheochromocytoma and paraganglioma; UCEC, uterine corpus endometrial carcinoma; ACC, adrenocortical carcinoma; OV, ovarian serous cystadenocarcinoma; CESC, cervical squamous cell carcinoma and endocervical adenocarcinoma; HNSC, head and neck squamous carcinoma; LIHC, liver hepatocellular carcinoma; THCA, thyroid carcinoma; BLCA, bladder urothelial carcinoma; PRAD, prostate adenocarcinoma; SKCM, skin cutaneous melanoma; STAD, stomach adenocarcinoma. 


\section{Declarations}

\section{Ethics approval and consent to participate}

The research didn't involve animal experiments and human specimens, no ethics related issues.

\section{Consent for publication}

Not applicable.

\section{Availability of data and materials}

All datasets were adopted in this study are available in TCGA (http://cancergenome.nih.gov/) database, Human Protein Atlas Datasets (https://www.proteinatlas.org/). and GEPIA2 (http://gepia2.cancerpku.cn/)database.

\section{Competing interests}

The authors declare no competing interests.

\section{Funding}

This work was funded by grants from the National Key R\&D Program (2017YFC0907400) and the Liaoning Provence Key R\&D Program (2020JH2/10300063).

\section{Authors' contributions}

LPS conceived and designed the research. COY and $\mathrm{HL}$ collected and analyzed the data. COY wrote the main manuscript text. LPS contributed to revising the manuscript. All authors read and approved the final manuscript.

\section{Corresponding author}

Correspondence to Liping Sun.

\section{Acknowledgements}

Not applicable.

\section{Authors' information}

Tumor Etiology and Screening Department of Cancer Institute and General Surgery, The First Hospital of China Medical University, No.155 NanjingBei Street, Heping District, Shenyang, Liaoning Province, P.R. China, 110001

Cheng Ouyang, Liping Sun 
Department of Clinical Laboratory, The First Hospital of China Medical University, No.155 NanjingBei Street, Heping District, Shenyang, Liaoning Province, P.R. China, 110001

Hao Li

\section{References}

1. You JS, Jones PA. Cancer genetics and epigenetics: two sides of the same coin? Cancer cell. 2012; 22(1).

2. Baylin SB, Jones PA. A decade of exploring the cancer epigenome - biological and translational implications. Nat Rev Cancer. 2011;11(10):726-34.

3. Verma M. Cancer control and prevention: nutrition and epigenetics. Curr Opin Clin Nutr Metab Care. 2013; 16(4):376-84.

4. Issa J-P. Cancer prevention: epigenetics steps up to the plate. Cancer Prev Res (Phila). 2008; 1(4):219-22.

5. Kelly AD, Issa J-PJ. The promise of epigenetic therapy: reprogramming the cancer epigenome. Curr Opin Genet Dev. 2017; 42:68-77.

6. Nowacka-Zawisza M, Wiśnik E. DNA methylation and histone modifications as epigenetic regulation in prostate cancer (Review). Oncology reports. 2017; 38(5):2587-96.

7. Suzuki MM, Bird A. DNA methylation landscapes: provocative insights from epigenomics. Nat Rev Genet. 2008; 9(6):465-76.

8. Pastor WA, Aravind L, Rao A. TETonic shift: biological roles of TET proteins in DNA demethylation and transcription. Nat Rev Mol Cell Biol. 2013; 14(6):341-56.

9. Shen L, Song C-X, He C, Zhang Y. Mechanism and function of oxidative reversal of DNA and RNA methylation. Annu Rev Biochem. 2014; 83:585-614.

10. Uysal F, Akkoyunlu G, Ozturk S. Dynamic expression of DNA methyltransferases (DNMTs) in oocytes and early embryos. Biochimie. 2015; 116:103-13.

11. Ito S, D'Alessio AC, Taranova OV, Hong K, Sowers LC, Zhang Y. Role of Tet proteins in $5 \mathrm{mC}$ to $5 \mathrm{hmC}$ conversion, ES-cell self-renewal and inner cell mass specification. Nature. 2010; 466(7310):1129-33.

12. Rasmussen KD, Helin K. Role of TET enzymes in DNA methylation, development, and cancer. Genes Dev. 2016; 30(7):733-50.

13. Uysal F, Ozturk S. DNA Methyltransferases in Mammalian Oocytes. Results and problems in cell differentiation. 2017; 63:211-22.

14. Zhang J, Yang C, Wu C, Cui W, Wang L. DNA Methyltransferases in Cancer: Biology, Paradox, Aberrations, and Targeted Therapy. Cancers (Basel). 2020; 12(8).

15. Gujar H, Weisenberger DJ, Liang G. The Roles of Human DNA Methyltransferases and Their Isoforms in Shaping the Epigenome. Genes. 2019; 10(2). 
16. Sun J, Fei F, Zhang M, Li Y, Zhang X, Zhu S, et al. The role of SEPT9 in screening, diagnosis, and recurrence monitoring of colorectal cancer. BMC cancer. 2019; 19(1):450.

17. Berman BP, Weisenberger DJ, Aman JF, Hinoue T, Ramjan Z, Liu Y, et al. Regions of focal DNA hypermethylation and long-range hypomethylation in colorectal cancer coincide with nuclear laminaassociated domains. Nature genetics. 2011; 44(1):40-6.

18. Jones PA, Baylin SB. The fundamental role of epigenetic events in cancer. Nature reviews Genetics. $2002 ; 3(6): 415-28$.

19. Esteller M. Epigenetics in cancer. N Engl J Med. 2008; 358(11):1148-59.

20. Moore LD, Le T, Fan G. DNA methylation and its basic function. Neuropsychopharmacology. 2013; 38(1):23-38.

21. Huang Y, Rao A. Connections between TET proteins and aberrant DNA modification in cancer. Trends in genetics : TIG. 2014; 30(10):464-74.

22. Li D, Bai Y, Feng Z, Li W, Yang C, Guo Y, et al. Study of Promoter Methylation Patterns of HOXA2, HOXA5, and HOXA6 and Its Clinicopathological Characteristics in Colorectal Cancer. Front Oncol. 2019; 9:394.

23. Song SJ, Poliseno L, Song MS, Ala U, Webster K, Ng C, et al. MicroRNA-antagonism regulates breast cancer stemness and metastasis via TET-family-dependent chromatin remodeling. Cell. 2013; 154(2):311-24.

24. Chen $\mathrm{C}$, Chen $\mathrm{H}$, Zhang $\mathrm{Y}$, Thomas HR, Frank MH, He Y, et al. TBtools: An Integrative Toolkit Developed for Interactive Analyses of Big Biological Data. Mol Plant. 2020; 13(8):1194-202.

25. Wang L-H, Huang J, Wu C-R, Huang L-Y, Cui J, Xing Z-Z, et al. Downregulation of miR-29b targets DNMT3b to suppress cellular apoptosis and enhance proliferation in pancreatic cancer. Molecular medicine reports. 2018; 17(2):2113-20.

26. Yang H, Liu Y, Bai F, Zhang JY, Ma SH, Liu J, et al. Tumor development is associated with decrease of TET gene expression and 5-methylcytosine hydroxylation. Oncogene. 2013; 32(5):663-9.

27. Tavakolian S, Goudarzi H, Faghihloo E. E-cadherin, Snail, ZEB-1, DNMT1, DNMT3A and DNMT3B expression in normal and breast cancer tissues. Acta Biochim Pol. 2019; 66(4):409-14.

28. Singal R, Das PM, Manoharan M, Reis IM, Schlesselman JJ. Polymorphisms in the DNA methyltransferase 3b gene and prostate cancer risk. Oncology reports. 2005; 14(2):569-73.

29. Afgar A, Fard-Esfahani P, Mehrtash A, Azadmanesh K, Khodarahmi F, Ghadir M, et al. MiR-339 and especially miR-766 reactivate the expression of tumor suppressor genes in colorectal cancer cell lines through DNA methyltransferase 3B gene inhibition. Cancer Biol Ther. 2016; 17(11):1126-38.

30. Kiehl S, Zimmermann T, Savai R, Pullamsetti SS, Seeger W, Bartkuhn M, et al. Epigenetic silencing of downstream genes mediated by tandem orientation in lung cancer. Scientific reports. 2017; 7(1):3896.

31. He M, Fan J, Jiang R, Tang W-X, Wang Z-W. Expression of DNMTs and MBD2 in GIST. Biomed Rep. 2013; $1(2): 223-7$. 
32. Cypris O, Božić T, Wagner W. Chicken or Egg: Is Clonal Hematopoiesis Primarily Caused by Genetic or Epigenetic Aberrations? Front Genet. 2019; 10:785.

33. Han M, Jia L, Lv W, Wang L, Cui W. Epigenetic Enzyme Mutations: Role in Tumorigenesis and Molecular Inhibitors. Front Oncol. 2019; 9:194.

34. Wang D, Huang J-H, Zeng Q-H, Gu C, Ding S, Lu J-Y, et al. Increased 5-hydroxymethylcytosine and Ten-eleven Translocation Protein Expression in Ultraviolet B-irradiated HaCaT Cells. Chin Med J (Engl). 2017; 130(5):594-9.

35. Delhommeau F, Dupont S, Della Valle V, James C, Trannoy S, Massé A, et al. Mutation in TET2 in myeloid cancers. N Engl J Med. 2009; 360(22):2289-301.

36. Ko M, Bandukwala HS, An J, Lamperti ED, Thompson EC, Hastie R, et al. Ten-Eleven-Translocation 2 (TET2) negatively regulates homeostasis and differentiation of hematopoietic stem cells in mice. Proc Natl Acad Sci U S A. 2011; 108(35):14566-71.

37. Quivoron C, Couronné L, Della Valle V, Lopez CK, Plo I, Wagner-Ballon O, et al. TET2 inactivation results in pleiotropic hematopoietic abnormalities in mouse and is a recurrent event during human lymphomagenesis. Cancer cell. 2011; 20(1):25-38.

38. Moran-Crusio K, Reavie L, Shih A, Abdel-Wahab O, Ndiaye-Lobry D, Lobry C, et al. Tet2 loss leads to increased hematopoietic stem cell self-renewal and myeloid transformation. Cancer cell. 2011; 20(1):11-24.

39. Dawson MA, Kouzarides T. Cancer epigenetics: from mechanism to therapy. Cell. 2012; 150(1):12-27.

40. Seshagiri S, Stawiski EW, Durinck S, Modrusan Z, Storm EE, Conboy CB, et al. Recurrent R-spondin fusions in colon cancer. Nature. 2012; 488(7413):660-4.

41. Sato Y, Yoshizato T, Shiraishi Y, Maekawa S, Okuno Y, Kamura T, et al. Integrated molecular analysis of clear-cell renal cell carcinoma. Nat Genet. 2013; 45(8):860-7.

42. Zhang Q, Huang R, Hu H, Yu L, Tang Q, Tao Y, et al. Integrative Analysis of Hypoxia-Associated Signature in Pan-Cancer. iScience. 2020; 23(9):101460.

43. Lian $\mathrm{H}$, Li W-B, Jin W-L. The emerging insights into catalytic or non-catalytic roles of TET proteins in tumors and neural development. Oncotarget. 2016; 7(39):64512-25.

44. D'Aniello C, Cermola F, Patriarca EJ, Minchiotti G. Vitamin C in Stem Cell Biology: Impact on Extracellular Matrix Homeostasis and Epigenetics. Stem Cells Int. 2017; 2017:8936156.

45. Pang AW, MacDonald JR, Pinto D, Wei J, Rafiq MA, Conrad DF, et al. Towards a comprehensive structural variation map of an individual human genome. Genome biology. 2010; 11(5):R52.

46. Zack TI, Schumacher SE, Carter SL, Cherniack AD, Saksena G, Tabak B, et al. Pan-cancer patterns of somatic copy number alteration. Nature genetics. 2013; 45(10):1134-40.

47. Lynch M. Genomics. Gene duplication and evolution. Science. 2002; 297(5583):945-7.

48. Yen RW, Vertino PM, Nelkin BD, Yu JJ, el-Deiry W, Cumaraswamy A, et al. Isolation and characterization of the cDNA encoding human DNA methyltransferase. Nucleic acids research. 1992; 20(9):2287-91. 
49. Kobayashi H, Sakurai T, Imai M, Takahashi N, Fukuda A, Yayoi O, et al. Contribution of intragenic DNA methylation in mouse gametic DNA methylomes to establish oocyte-specific heritable marks. PLoS Genet. 2012; 8(1):e1002440.

50. Li Y, Zhang Z, Chen J, Liu W, Lai W, Liu B, et al. Stella safeguards the oocyte methylome by preventing de novo methylation mediated by DNMT1. Nature. 2018; 564(7734):136-40.

51. Inoue A, Zhang Y. Replication-dependent loss of 5-hydroxymethylcytosine in mouse preimplantation embryos. Science. 2011; 334(6053):194.

52. Yang R, Qu C, Zhou Y, Konkel JE, Shi S, Liu Y, et al. Hydrogen Sulfide Promotes Tet1- and Tet2Mediated Foxp3 Demethylation to Drive Regulatory T Cell Differentiation and Maintain Immune Homeostasis. Immunity. 2015; 43(2):251-63.

53. Yue X, Lio C-WJ, Samaniego-Castruita D, Li X, Rao A. Loss of TET2 and TET3 in regulatory T cells unleashes effector function. Nature communications. 2019; 10(1):2011.

54. Ichiyama K, Chen T, Wang X, Yan X, Kim B-S, Tanaka S, et al. The methylcytosine dioxygenase Tet2 promotes DNA demethylation and activation of cytokine gene expression in T cells. Immunity. 2015; 42(4):613-26.

55. Shen Y, Zhu Y-M, Fan X, Shi J-Y, Wang Q-R, Yan X-J, et al. Gene mutation patterns and their prognostic impact in a cohort of 1185 patients with acute myeloid leukemia. Blood. 2011; 118(20):5593-603.

56. Cao X-Y, Ma H-X, Shang Y-H, Jin M-S, Kong F, Jia Z-F, et al. DNA methyltransferase3a expression is an independent poor prognostic indicator in gastric cancer. World J Gastroenterol. 2014; 20(25):8201-8.

57. Gao Q, Steine EJ, Barrasa MI, Hockemeyer D, Pawlak M, Fu D, et al. Deletion of the de novo DNA methyltransferase Dnmt3a promotes lung tumor progression. Proceedings of the National Academy of Sciences of the United States of America. 2011; 108(44):18061-6.

58. Szablewski V, Bret C, Kassambara A, Devin J, Cartron G, Costes-Martineau V, et al. An epigenetic regulator-related score (EpiScore) predicts survival in patients with diffuse large B cell lymphoma and identifies patients who may benefit from epigenetic therapy. Oncotarget. 2018; 9(27):19079-99.

59. Patnaik MM, Barraco D, Lasho TL, Finke CM, Hanson CA, Ketterling RP, et al. DNMT3A mutations are associated with inferior overall and leukemia-free survival in chronic myelomonocytic leukemia. $A m$ J Hematol. 2017; 92(1):56-61.

60. Belani R, Oliveira G, Erikson GA, Ra S, Schechter MS, Lee JK, et al. ASXL1 and DNMT3A mutation in a cytogenetically normal B3 thymoma. Oncogenesis. 2014; 3:e111.

\section{Figures}


(a)

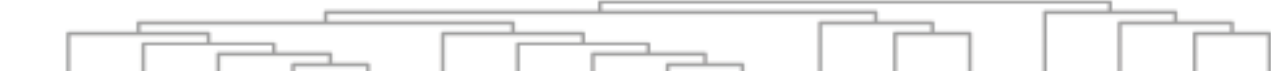

\begin{tabular}{|l|l|l|l|l|l|l|l|l|l|l|l|l|l|l|l|l|l|l|}
\hline 2.63 & 2.70 & 1.34 & 2.08 & 2.44 & 2.49 & 0.69 & 1.31 & 1.82 & 1.79 & -1.20 & 0.33 & 0.41 & -7.32 & -4.98 & -4.04 & -3.01 & DNMT3L & DNT \\
\hline
\end{tabular}

\begin{tabular}{|l|l|l|l|l|l|l|l|l|l|l|l|l|l|l|l|l|l}
2.72 & 2.04 & 2.35 & 1.60 & 2.26 & 2.68 & 2.06 & 1.50 & 2.20 & 2.32 & 1.68 & 0.57 & -0.33 & 1.56 & -0.10 & 1.52 & 0.57 & DNMT3B \\
\hline
\end{tabular}

\begin{tabular}{|l|l|l|l|l|l|l|l|l|l|l|l|l|l|l|l|l|}
\hline 0.04 & -0.40 & 0.05 & 0.11 & 0.07 & -0.70 & -0.38 & -0.55 & -0.39 & -0.21 & -0.16 & 0.08 & -0.39 & 0.76 & -1.55 & -0.46 & -0.01 \\
\hline
\end{tabular}





\begin{tabular}{|l|l|l|l|l|l|l|l|l|l|l|l|l|l|l|l|l}
0.52 & -0.43 & -0.03 & 0.69 & 0.58 & 1.90 & 1.57 & 0.04 & 0.45 & 0.57 & 1.42 & 0.11 & -0.21 & 1.27 & -1.62 & -0.29 & -0.11 \\
\hline
\end{tabular}

0.50

0.00

$-0.50$

\begin{tabular}{|l|l|l|l|l|l|l|l|l|l|l|l|l|l|}
0.88 & 0.55 & 1.41 & 0.94 & 1.69 & 0.52 & -0.21 & 0.13 & -0.30 & 0.23 & -0.32 & 0.30 & 0.08 \\
\hline
\end{tabular}

\begin{tabular}{l|l|l|l|l}
0.36 & 0.46 & 0.88 & TET1P1
\end{tabular}

\begin{tabular}{|l|l|l|l|l|l|l|l|l|l|l|l|l|l|l|l|l|l}
1.44 & 0.92 & 1.10 & 0.90 & 1.21 & 0.00 & 0.65 & 1.01 & 1.03 & 0.91 & 1.16 & 0.09 & 0.57 & 1.16 & 0.36 & 0.23 & 0.37 & DNMT1
\end{tabular}

\begin{tabular}{|l|l|l|l|l|l|l|l|l|l|l|l|l|l|l|l|l|l}
0.87 & 0.32 & 0.19 & 0.26 & -0.13 & 1.08 & 0.91 & 0.75 & 0.69 & 0.25 & 1.29 & 0.43 & 0.06 & 1.63 & -0.32 & 0.25 & 0.28 & DNMT3A
\end{tabular}

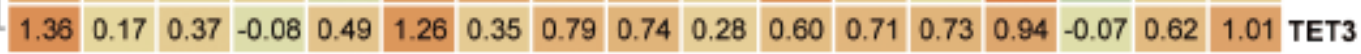

岁 焉

(b)


Figure 1

DNMT/TET genes mRNA expression profiles across different cancer types. (a) DNMT/TET genes expression in different cancers and normal samples. The color in heatmap represents the log2 fold change value between cancer and normal. The blue color represents low expression in cancer while the red color represents high expression in cancer. (b) DNMT3B expression in 16 cancer types in cancer and normal tissues. 
(a)

Protein Expression
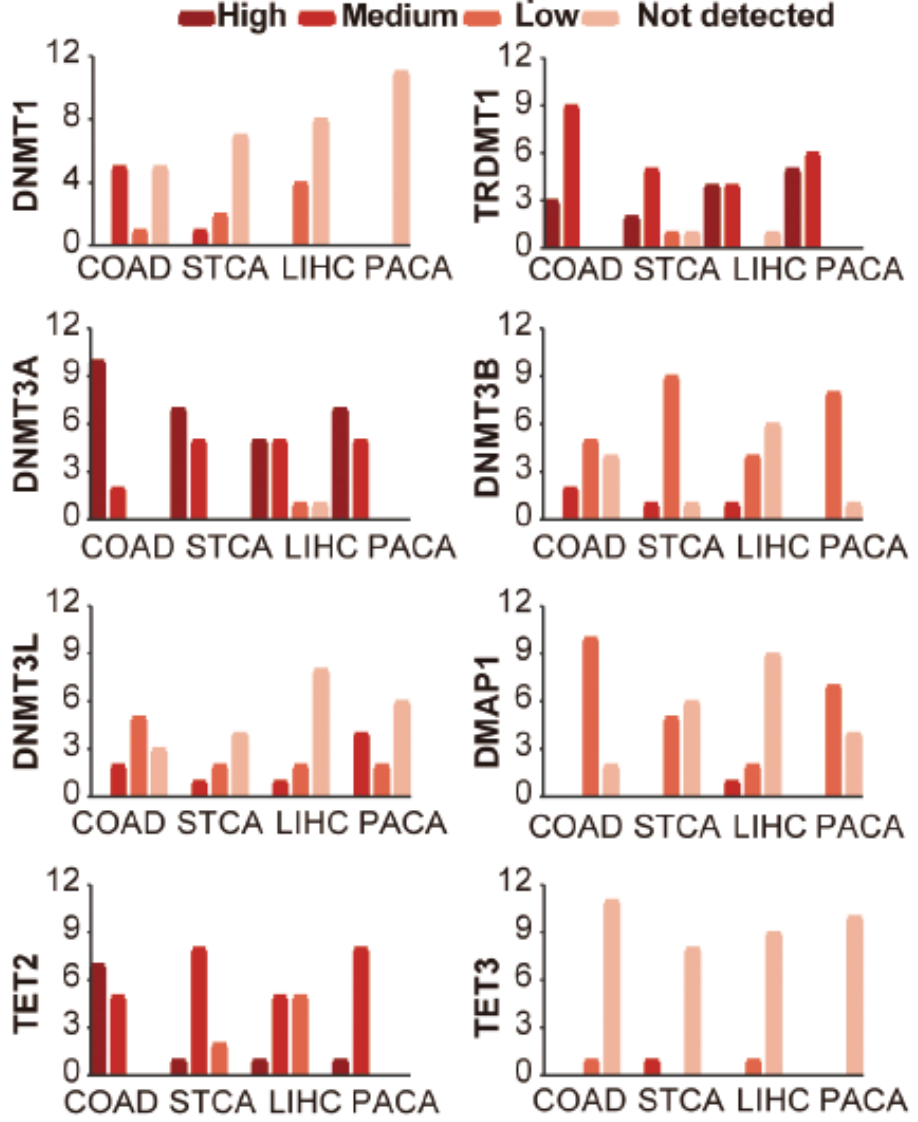

(b)
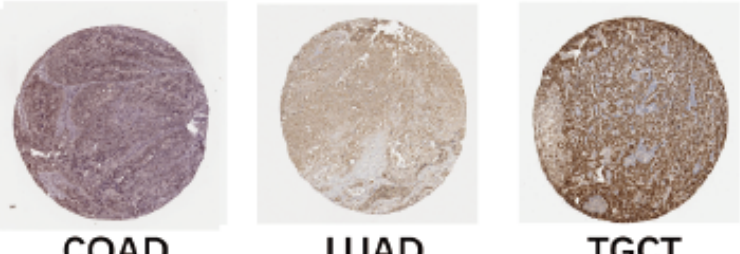

LUAD
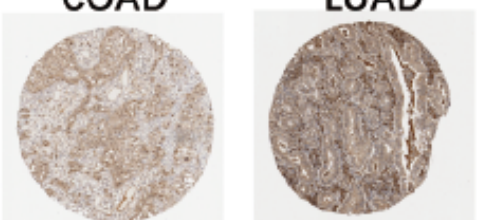

TGCT

HNSC

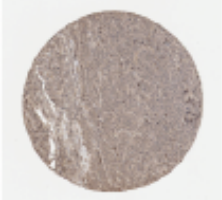

LIHC

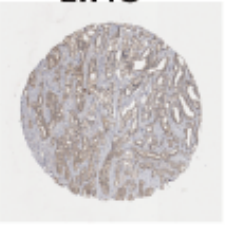

PRAD

STAD

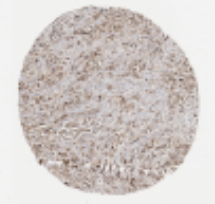

PAAD

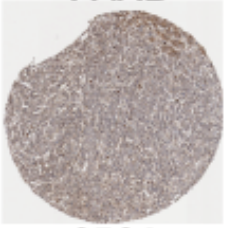

CECA

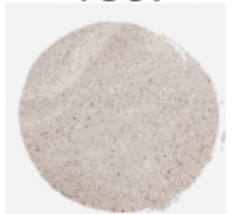

Glioma

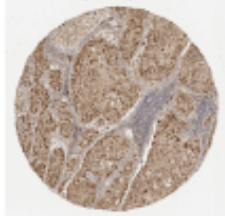

URCA



UCEC

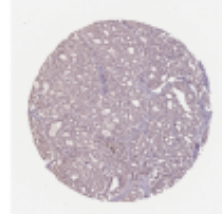

THCA

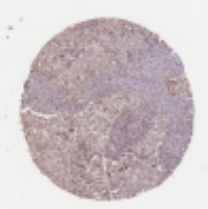

BRCA

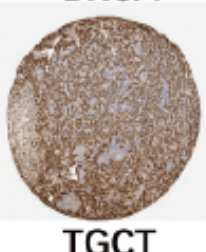

TGCT

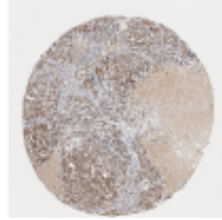

OV

Figure 2

DNMT/TET protein expression profiles across different cancer types. (a) DNMT/TET protein expression across different cancers. In one cancer gene expression was divided into four groups: high expression, medium expression, low expression, and not detected. (b) DNMT3B protein expression in 16 cancer types based on immunohistochemistry staining. 


\section{corr \\ $\begin{array}{lllll}-1.00 & -0.50 & 0.00 & 0.50 & 1.00\end{array}$}

DMAP1 DNMT1 TRDMT1 TET2 DNMT3B DNMT3L TET1

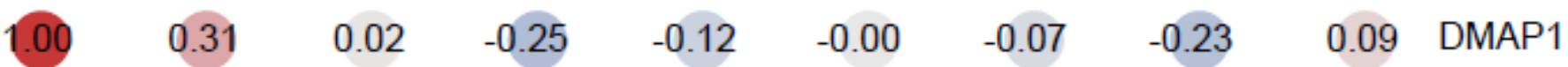

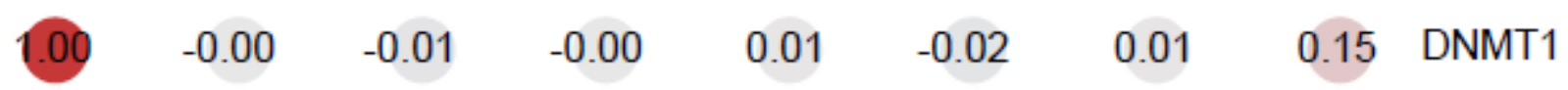

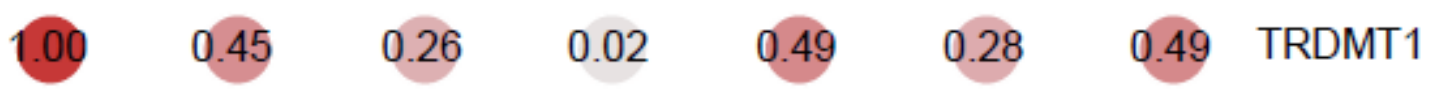

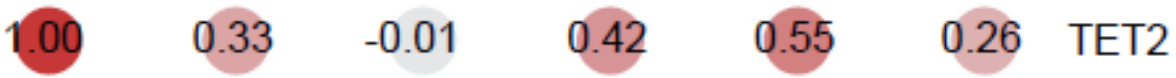

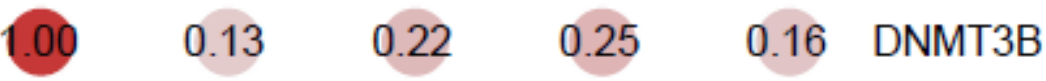

$1.00 \quad 0.03 \quad-0.05 \quad 0.01 \quad$ DNMT3L

$000.50 \quad 0.57 \quad$ TET1

000.48 TET3

\section{Figure 3}

Correlation between DNMT/TET genes expression. 
(a)

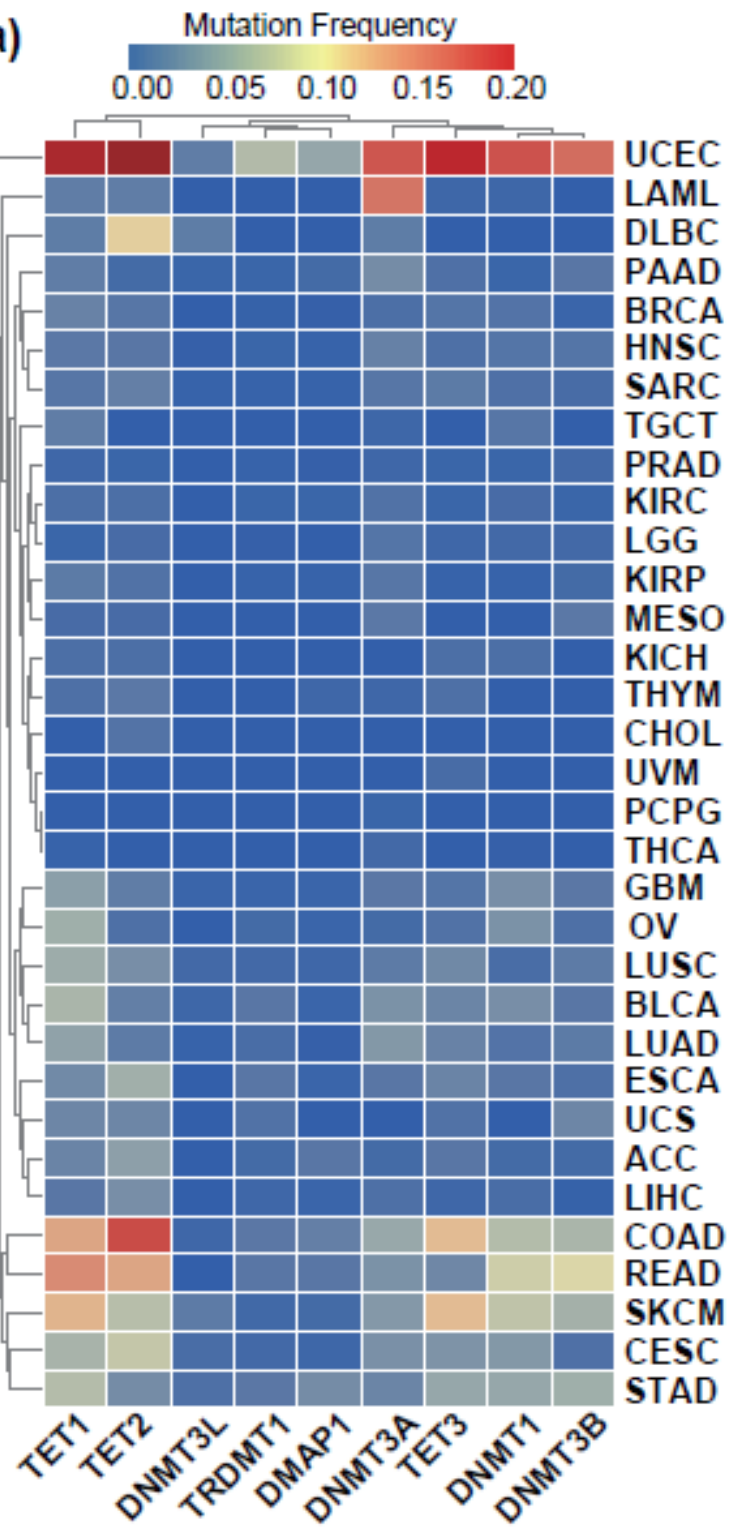

$(b-1)$

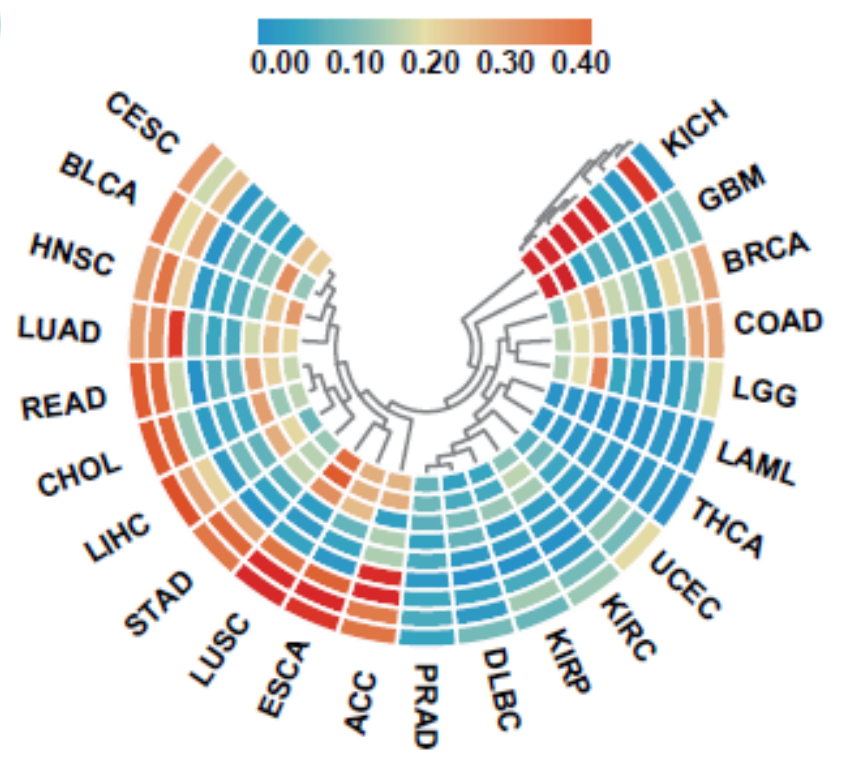

(b-2)

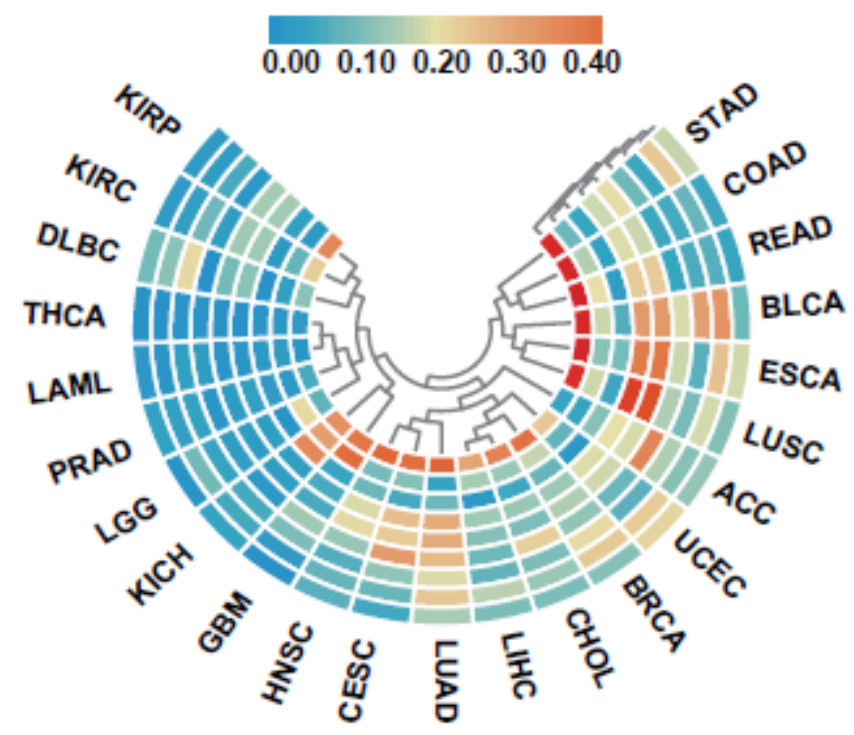

Figure 4

Pan-cancer genetic alterations in DNMT/TET genes. (a) DNMT/TET genes pan-cancer mutation frequency. (b-1) DNMT/TET genes copy number amplification, each loop from the outside to the inside represents a gene (TET1, TRDMT1, DNMT3L, DMAP1, TET3, DNMT3A, TET2, DNMT1, and DNMT3B). (b2) DNMT/TET genes copy number deletion, each loop from the outside to the inside represents a gene (TET2, DNMT3L, DNMT1, DNMT3B, TET3, DNMT3A, DMAP1, TET1, and TRDMT1) 

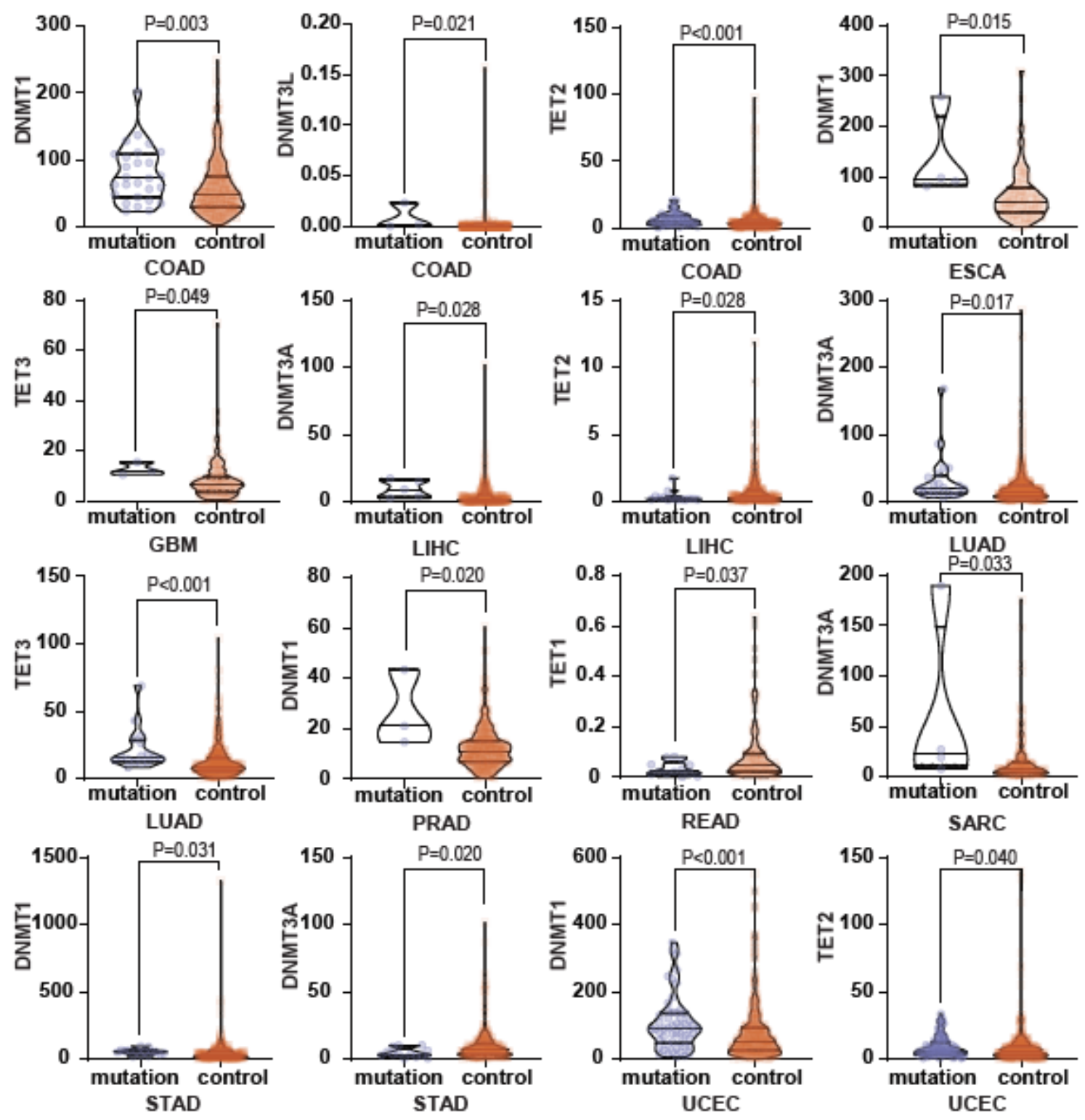

\section{Figure 5}

The association between DNMT/TET genes expression levels and mutation frequency in pan-cancer. 
Deletion Normal Amplification
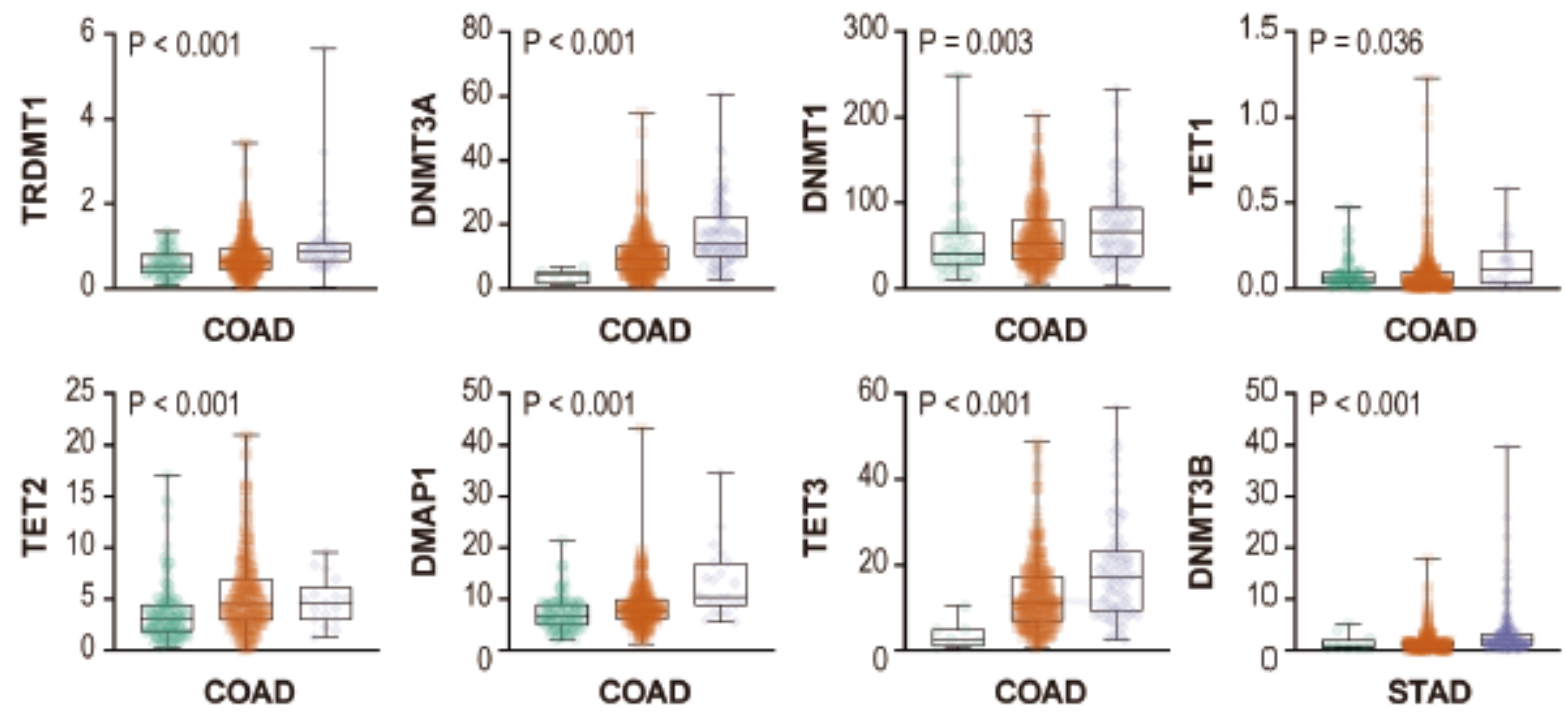

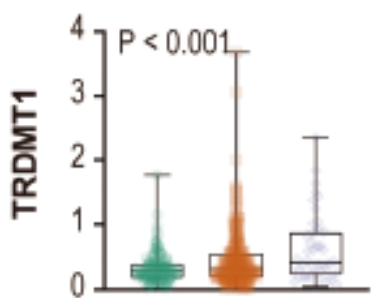

STAD

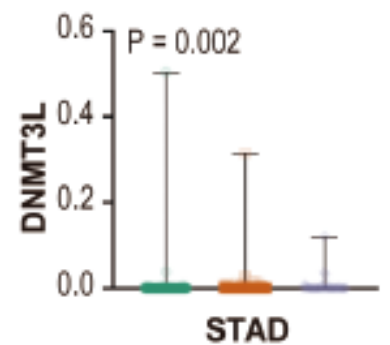

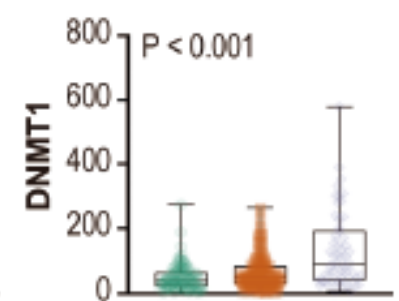

STAD

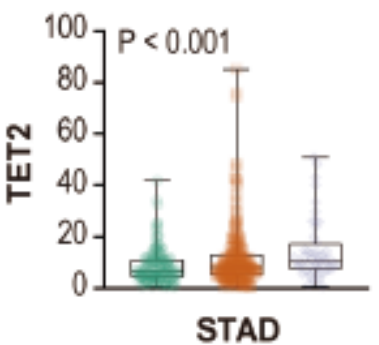

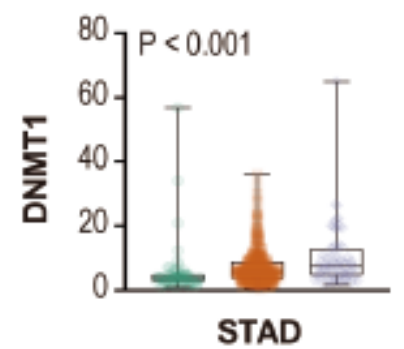

STAD

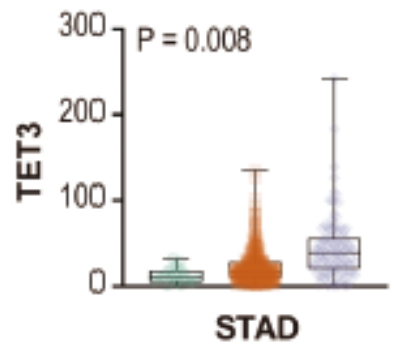

Figure 6

The association between DNMT/TET genes expression levels and copy number variations in different cancers. 
(a)



(b)

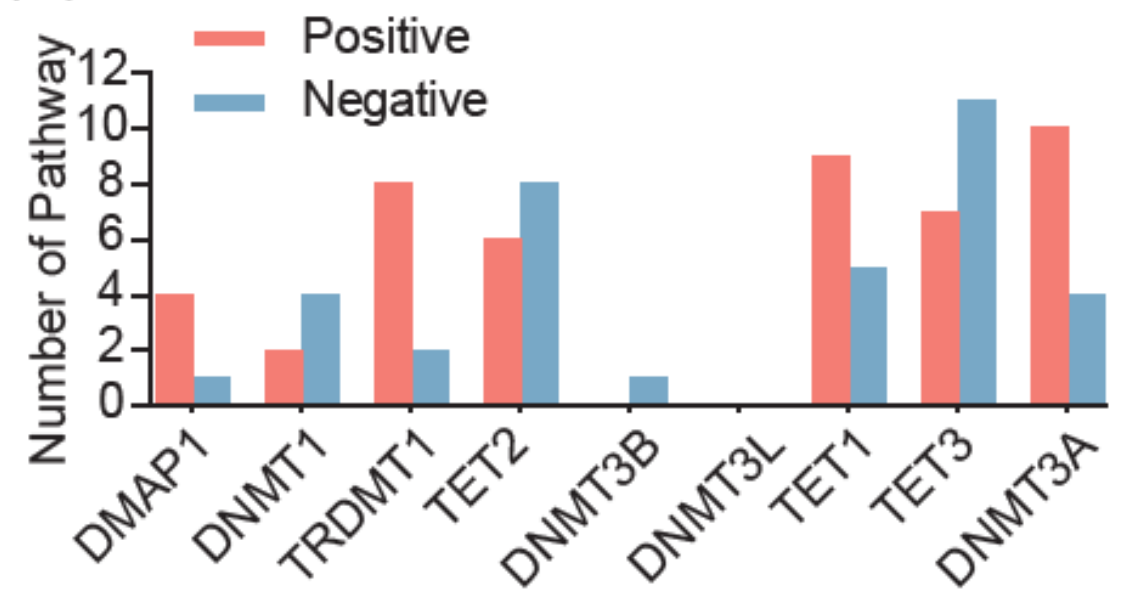

Figure 7

Association between DNMT/TET genes and cancer-related pathways. (a) Network diagram demonstrating the correlation between DNMT/TET genes and cancer-related pathways. Green nodes represent negative correlation pathways while red nodes represent positive pathways. (b) The number of correlated pathways in individual DNMT/TET genes. 


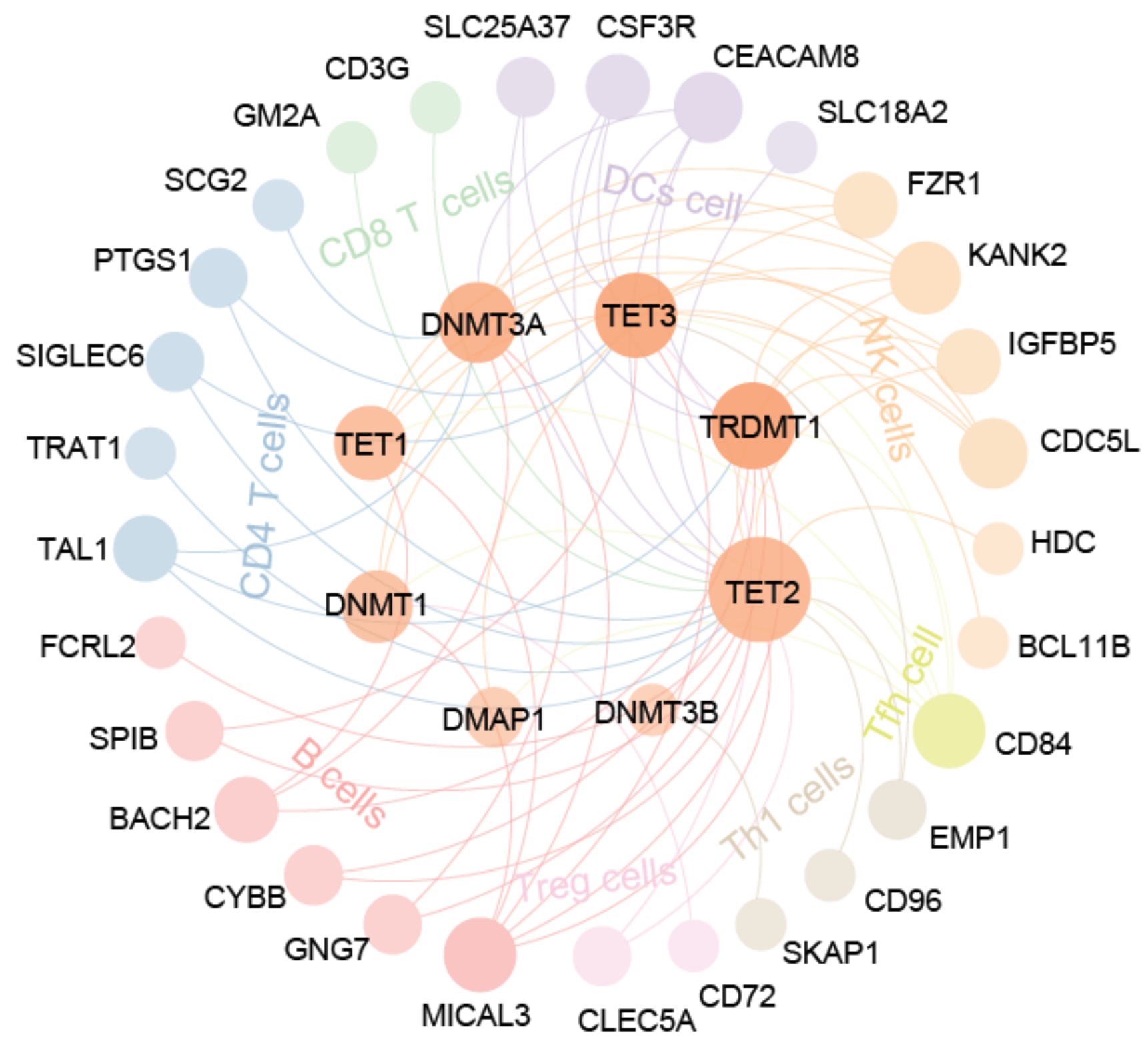

Figure 8

Correlation between DNMT/TET genes and immune cell infiltration. The genes in the outer circle represent genes within individual immune cells. Inner circles are formed by DNMT/TET genes. The size of each gene represents the number of connections. 
(a)

High risk Low risk $\mathrm{P}>=0.05$

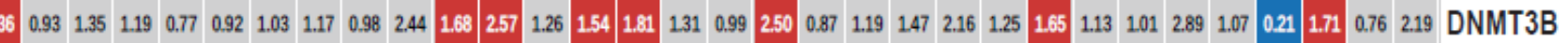

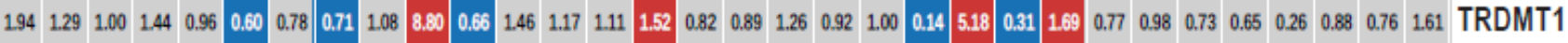

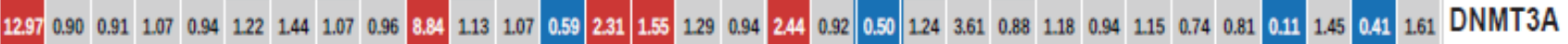

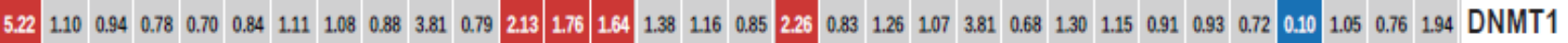

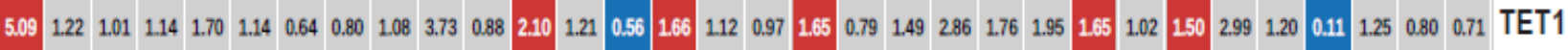

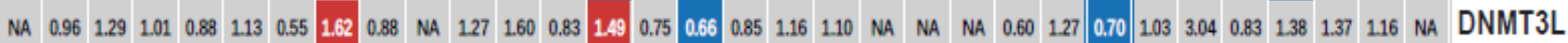

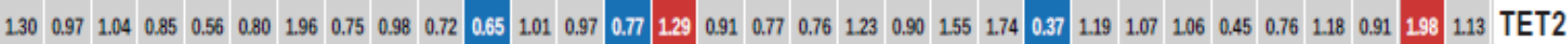

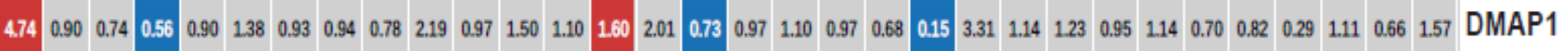

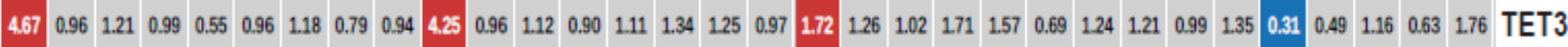

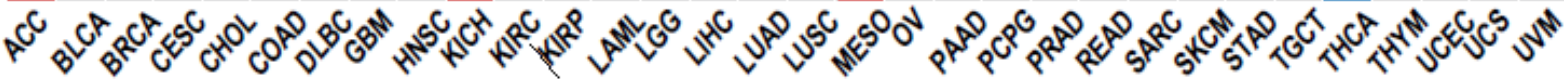

(b)

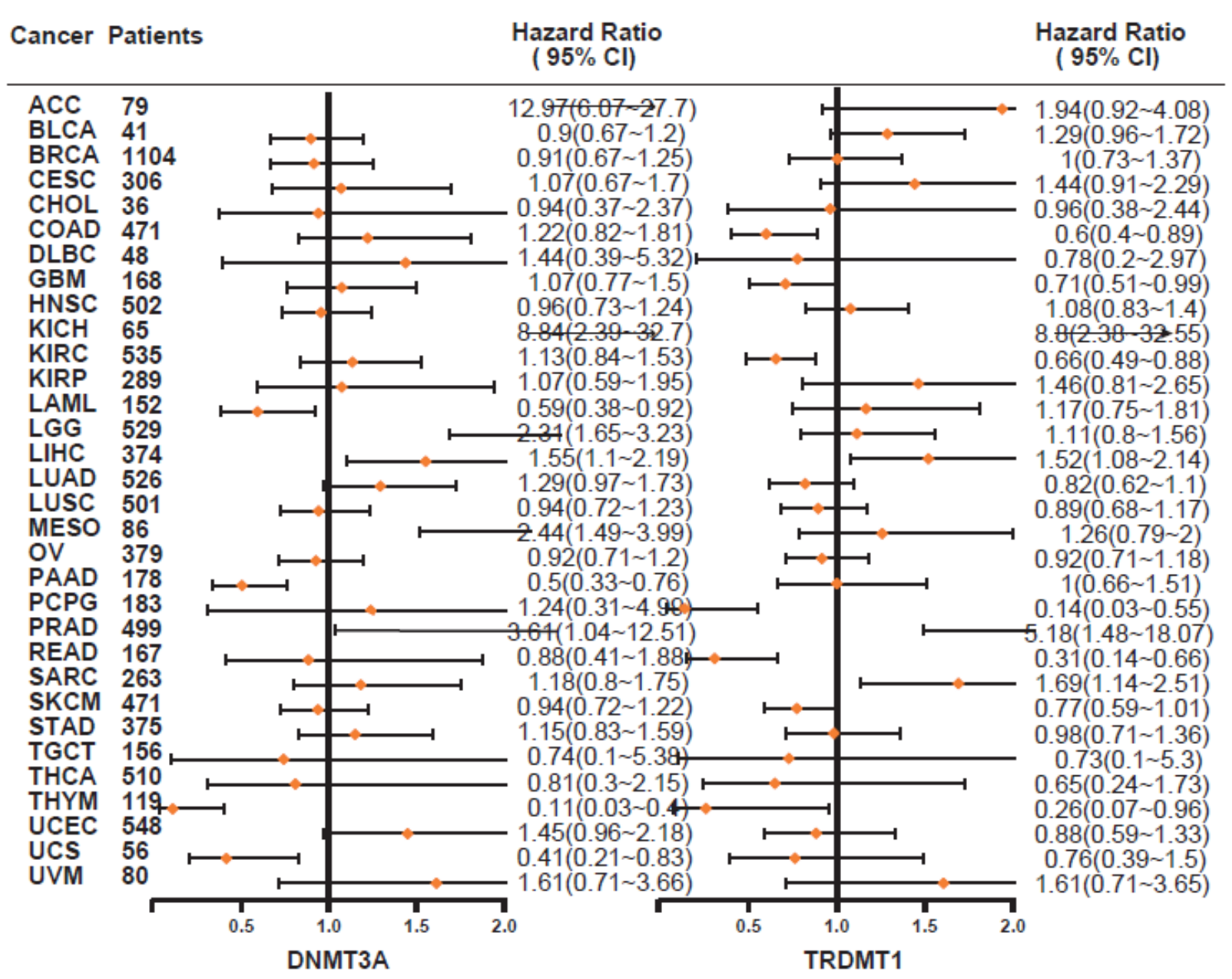

Figure 9

Prognostic significance of DNMT/TET genes. (a) Summary of the correlation between DNMT/TET genes expression and survival in different cancers. Red color represents high risk of death and blue color represents low risk of death. (b) Forest plot for DNMT3A and TRDMT1 prognostic analysis across various cancer types. 

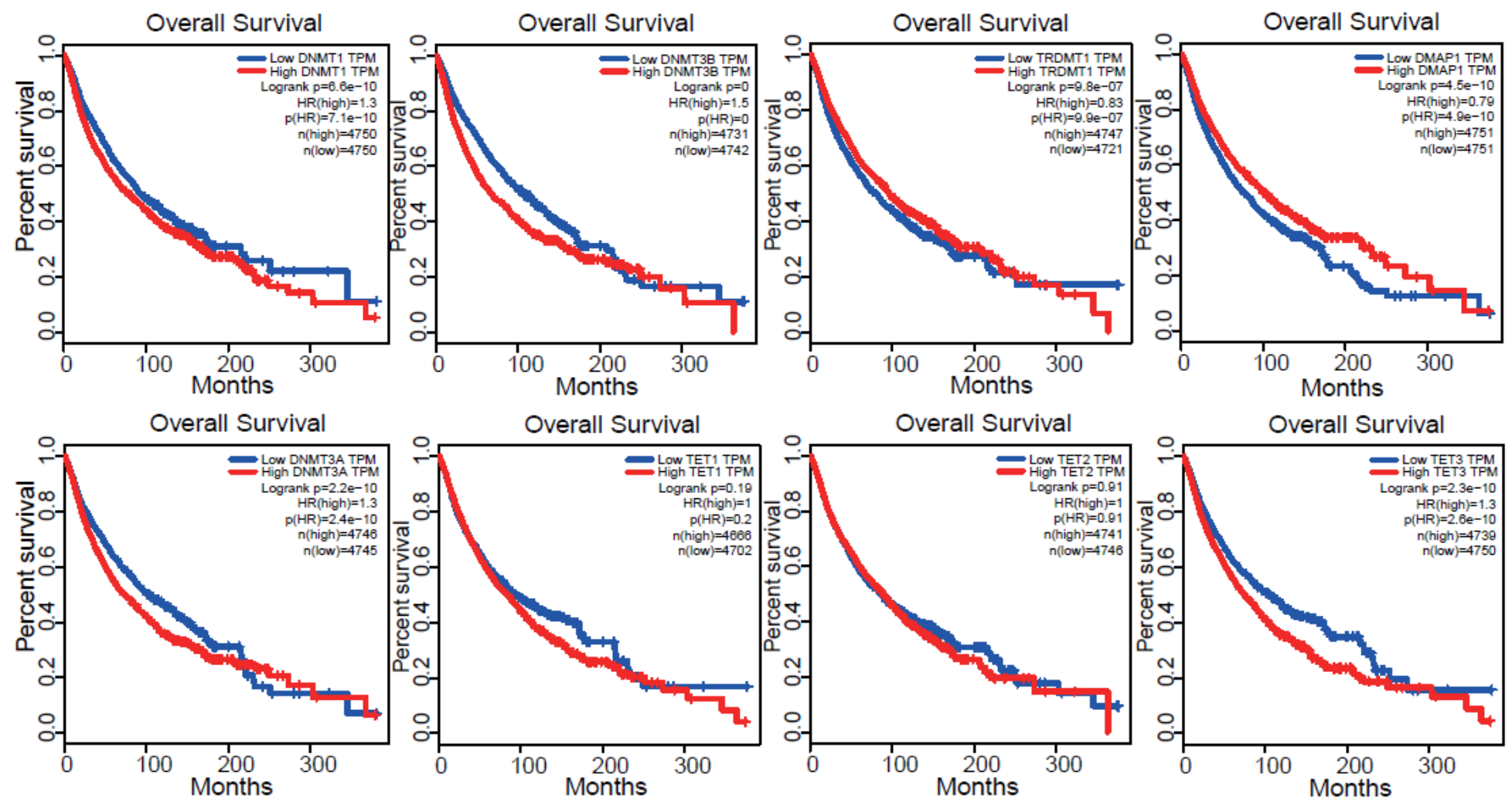

Figure 10

Survival analysis for DNMT/TET genes in pan-cancer. The median expression level was selected as the cut-off value.

\section{Supplementary Files}

This is a list of supplementary files associated with this preprint. Click to download.

- SupplementaryTable1.xls

- SupplementaryTable2.xIsx

- SupplementaryTable3.xlsx

- SupplementaryTable4.xlsx 